\title{
Comparative Evaluation of Mortality and Hemodynamic Performance Between ATS and ST JUDE Mechanical Heart Valves in Mitral and Aortic Positions
}

\author{
Mohammad Rafi Hamidi ${ }^{1}$, Manizha Meena ${ }^{2}$, Mezhgan Zaher ${ }^{2}$, Mohammad Hussain Shiwa ${ }^{2}$, \\ Abdul Wahid Hussaini ${ }^{2}$, Assadullah Hassani ${ }^{3}$, Manochihr Timorian ${ }^{4, *}$ \\ ${ }^{1}$ Deputy ARCS Health, Senior Fellow Department of Cardiothoracic and Vascular Surgery Global Medical Complex Heart Institute, Kabul, \\ Afghanistan \\ ${ }^{2}$ Fellows Department of Cardiothoracic and Vascular Surgery; Global Medical Complex Heart Institute, Kabul, Afghanistan \\ ${ }^{3}$ Head Department of Cardiology, Global Medical Complex Heart Institute, Kabul, Afghanistan \\ ${ }^{4}$ Head Department of Cardiothoracic and Vascular Surgery Global Medical Complex Heart Institute, Kabul, Afghanistan
}

Email address:

manochihr@yahoo.com (M. Timorian)

${ }^{*}$ Corresponding author

\section{To cite this article:}

Mohammad Rafi Hamidi, Manizha Meena, Mezhgan Zaher, Mohammad Hussain Shiwa, Abdul Wahid Hussaini, Assadullah Hassani, Manochihr Timorian. Comparative Evaluation of Mortality and Hemodynamic Performance Between ATS and ST JUDE Mechanical Heart Valves in Mitral and Aortic Positions. International Journal of Cardiovascular and Thoracic Surgery. Vol. 6, No. 6, 2020 , pp. 66-69. doi: $10.11648 /$ j.ijcts.20200606.11

Received: October 15, 2020; Accepted: October 26, 2020; Published: November 19, 2020

\begin{abstract}
Background: Rheumatic heart disease is the most common heart disease in Asian countries especially in Afghanistan, the age adjusted death rate for this heart disease is 27,57 per 100000 people as published by data of the World Health Organization (WHO). ST JUDE mechanical heart valve first time implanted in October 1977 and quickly became the gold standard for subsequent valves, and ATS medical international developed a mechanical heart valve that has been in use since 1992, these mechanical heart valves started implantation in Afghanistan during 2012. We presented a result of 148 patients who have undergone valve replacement in mitral and aortic position with ATS and ST JUDE mechanical heart valves at departments of cardiothoracic and vascular surgery amiri medical complex and global medical complex heart institute Kabul Afghanistan. Method and results: we performed ATS and ST JUDE mechanical heart valve replacement in 148 patients between May 2015 and April 2018 at both the Department of Cardiothoracic and Vascular Surgery Global Medical Complex heart institute and Amiri Medical Complex, Kabul Afghanistan. Male patients were $69(46.6 \%)$ and female patients were $79(53.3 \%)$, age range was between 11-65 years, $94(63.5 \%)$ patients under went mitral valve replacement, for $38(25.6 \%)$ patients performed aortic valve replacement and $16(10.8 \%)$ patients required double valve replacement, overall mortality was $16(10.8 \%)$ patients for mitral, aortic and double valve replacement. The early mortality (hospital mortality) was $4.05 \%$ and late mortality during a 3 year follow up was $6.7 \%$. Conclusion: there were seen a few prosthetic valve complications after ATS and ST JUDE mechanical heart valve implantation (total number of implanted ATS mechanical heart valve were 70 and total number of implanted ST JUDE mechanical heart valve were 78), early mortality was (hospital mortality) 6 patients (4.05\%) and late mortality during a 3 year follow up was 10 patients $(6.7 \%)$. The international normalized ratio (INR) was maintained between (2.5-3.5) in both ATS and ST JUDE mechanical heart valves for mitral position and (2-3) for aortic position, hemodynamically ATS and ST JUDE mechanical heart valves are very good regarding trans valvular gradient and function but low prosthetic valve noise is just seen in ATS mechanical heart valve.
\end{abstract}

Keywords: Cardiac Surgery, Valve Surgery, Mitral and Aortic Valve Replacement 


\section{Introduction}

Rheumatic heart disease is the most common cause of valvular heart disease in Asian countries specially in Afghanistan, the age adjusted death rate for this heart disease is 2757 per 100000 people as published by data of the World Health Organization (WHO). Most heart valve replacements were due to rheumatic heart diseases and a smaller number of valves replaced due to infective endocarditis, mechanical heart valve implantation started in Afghanistan since 2012.

\subsection{Patients and Method}

Between May 2015 and April 2018, 148 patients undergone valve replacement with ATS and ST JUDE mechanical heart valves in mitral and aortic positions at Departments of Cardiothoracic and Vascular Surgery Global Medical Complex Heart institute and Amiri Medical Complex, Kabul Afghanistan.

This study includes $69(46.6 \%)$ male patients and 79 (53.3\%) female patients, age range was between 11-65 years. $94(63.5 \%)$ patients underwent mitral valve replacement (48 patients with ATS and 46 patients with ST JUDE mechanical heart valves), for $38(25.6 \%)$ patients performed aortic valve replacement (16 patients with ATS and 22 patients with ST JUDE mechanical heart valves). Patients who underwent double valve replacement (mitral and aortic) were $16(10.8 \%)$ patients (6 patients with ATS and 10 patients with ST JUDE mechanical heart valves) table 1.

Table 1. Total number of mechanical valves implanted in aortic and mitral position (148).

\begin{tabular}{llll}
\hline Valve position & Total number & ATS mechanical valve & ST JUDE mechanical valve \\
\hline Mitral & 94 & 48 & 46 \\
Aortic & 38 & 16 & 22 \\
Mitral and aortic (double valve replacement) & 16 & 6 & 10 \\
\hline
\end{tabular}

Regarding New York Heart Association (NYHA) symptoms classification, $33(22.2 \%)$ patients were in NYHA class II, $65(43.9 \%)$ patients were in (NYHA) class III, and $50(33.7 \%)$ patients were in class IV. Atrial fibrillation presented in $88(59.4 \%)$ patients with mitral valve diseases and $22(14.8 \%)$ patients had left atrial clot. There were 2 $(1.35 \%)$ patients with mitral valve diseases and ischemic stroke in the preoperative period. In addition, 5 patients underwent mitral valve replacement concomitant with coronary artery bypass grafting surgery and 2 patients had double valve replacement with coronary artery bypass grafting surgery and $55(37,1 \%)$ patients had concomitant tricuspid valve repair (devega suture annuloplasty) with mitral valve replacement.

Etiology of valve replacement included rheumatismal valvular heart disease in $90 \%$ of patients, degenerative valvular heart disease in $5 \%$ of patients and $5 \%$ patients with infective endocarditis.

Table 2. Patients demographic.

\begin{tabular}{|c|c|c|c|c|c|c|c|c|}
\hline & \multirow[b]{2}{*}{ Parameter } & \multicolumn{3}{|c|}{ Single valve procedure } & \multicolumn{4}{|c|}{ double valve procedure } \\
\hline & & MVR & MVR+TVR & MVR+CABG & AVR & DVR & DVR+CABG & TOTAL \\
\hline Patients (n) & & & 55 & 5 & 38 & 14 & 2 & $148(100 \%)$ \\
\hline \multirow[t]{4}{*}{ Preoperative NYHA } & & 34 & & & & & & \\
\hline & II & & 20 & - & 10 & - & - & $33(22,2 \%)$ \\
\hline & III & 3 & 22 & 2 & 6 & 122 & $65(43,9 \%)$ & \\
\hline & IV & 21 & 13 & 3 & 22 & 2 & - & $50(33,7 \%)$ \\
\hline Preoperative AF & & 10 & 49 & 3 & - & 10 & 2 & $88(59,4 \%)$ \\
\hline Preoperative stroke & & 810 & 1 & - & -- & & & $2(1,35 \%)$ \\
\hline
\end{tabular}

$\mathrm{MVR}=$ Mitral valve replacement, $\mathrm{TVR}=$ Tricuspid valve repair, $\mathrm{AVR}=$ Aortic valve replacement, $\mathrm{DVR}=\mathrm{Double}$ valve replacement, $\mathrm{CABG}=\mathrm{Coronary}$ artery bypass grafting, $\mathrm{NYHA}=\mathrm{New}$ York heart association, $\mathrm{AF}=$ Atrial fibrillation, $\mathrm{LA}=\mathrm{Left}$ atrium

\subsection{Surgical Procedure}

Patients were operated on through a median sternotomy with mild to moderate hypothermia and cardiopulmonary bypass, myocardial preservation was made by antigrade cold cardioplegia, yet in the majority of cases (mitral valve replacement) the native posterior leaflet of mitral valve was not excised for preserving of left ventricle function, the valve annulus was measured with ATS and STJ valve sizers and valves were fixed with interrupted horizontal mattress sutures used 2.0 ethibond (ethicon, Int, a Johnson \& Johnson company).

\subsection{Postoperative Care}

Postoperative recovery was conducted in the intensive care unit (ICU) until the patients were weaned off both ventilator and inotropic support, patients were then transferred to a ward after 2-3 days. On the second postoperative day the drainage tubes were removed and oral anticoagulant acitrom (acenocoumarol/nicoumalone) or warfarin was initiated with target international normalize ratio (INR) of 2-3 for aortic valve replacement and 2.5-3.5 for mitral valve and double valve (mitral plus aortic) replacement in addition to $75 \mathrm{mg}$ of aspirin added as a daily antiplatelet therapy.

Echocardiographic monitoring was performed between the $5^{\text {th }}$ and $7^{\text {th }}$ postoperative days, with the valve gradient calculated by means of the modified Bernoulli equation. Valve size distribution and associated postoperative gradients are reported for both aortic and mitral valves in tables 3 and 4 . 
Table 3. Valve size distribution and postoperative mean gradient in mitral valve position.

\begin{tabular}{lll}
\hline Valve size & number & mean gradient \\
\hline 25 & 10 & $6.5 \pm 2.2$ \\
27 & 24 & $4.6 \pm 2.1$ \\
29 & 30 & $4.1 \pm 1.7$ \\
31 & 18 & $3.8 \pm 1.6$ \\
33 & 12 & $4.0 \pm 1.2$ \\
\hline
\end{tabular}

Table 4. Valve size distribution and postoperative mean gradient in aortic valve position.

\begin{tabular}{lll}
\hline Valve size & number & mean gradient \\
\hline 18 & 2 & $19 \pm 1.2$ \\
19 & 6 & $19 \pm 1.4$ \\
21 & 20 & $12 \pm 1.0$ \\
23 & 8 & $9 \pm 2.3$ \\
25 & 2 & $8 \pm 1.3$ \\
\hline
\end{tabular}

\subsection{Patients Follow up}

After hospital discharge long term anticoagulation management was under direction of a local general practitioner to maintain INR between 2-3 in aortic position and 2.5-3.5 in mitral position. Echocardiographic follow up was performed postoperatively at 3 months, 6 months and annually thereafter by a cardiologist. All patients were followed up annually in the outpatient clinic by operative cardiac surgeons, the data collection protocol required contacting both the patients and the patients following physician, also, patients were asked to report adverse events and then the most recent INR measurement and self-reported adverse events were verified with the appropriate following physician.

\section{Result}

Early postoperative mortality and morbidity:

Mean ICU (Intensive Care Unit) stay was 2-4 days and hospital stay was 5-12 days in all valve groups (ATS and ST jude mechanical heart valve), early mortality was 6 patients (during 30 days of postoperative) and only 3 patients died because of acute left ventricle dysfunction, 2 patients died due to sepsis and 1 patient because of multiorgan failure. There was no on table death.

We had 2 patients with severe pericardial effusion who were treated by pericardial synthesis under cathlab fluoroscopy, there were 2 patients with perioperative ischemic stroke mainly with mitral valve disease and had just two patients with postoperative stroke, one patient after aortic valve replacement with aortic root suture line dehiscence and another after double valve replacement with coronary artery bypass grafting surgery (CABG) due to hypertensive brain hemorrhage. Echocardiographic assessment revealed a very low mean gradient pressure by valve size. (Tables 3 and 4).

Late valve related complications:

1. Thromboembolic event:

There were 6 patients with thromboembolic events, 4 for ST Jude and 2 for ATS groups, 4 of them had neurologic consequences and 2 of them had reversible ischemic neurological defect.

2. Bleeding events:

A total of 4 bleeding events were seen in both (2 ATS and 2 ST jude) groups with 3 massive intraperitoneal and pericardial bleeding that required hospitalization and 2 of them died due to multiorgan failure.

We had just one patient with minor bleeding, this anticoagulant related hemorrhage did not require hospitalization.

3. other complications:

There was no valvular and para valvular leak in either ST Jude and ATS mechanical heart valve groups, with just one case of stuck valve in mitral valve position of ST Jude group, who underwent reoperation.

There was no structural valve deterioration detected in ATS and ST Jude mechanical heart valve groups.

New York Heart Association (NHA) functional classification symptomatic improvement occurred in all patients after valve replacement in both ATS and ST Jude groups.

\section{Discussion}

The main disadvantage of mechanical heart valves are their thromboembolic tendencies and the patients need to use anticoagulants for life with the related risk of hemorrhage [4, $6]$.

To achieve perfect performance a prosthetic valve should possess good hemodynamic performance and durability, ease of insertion and freedom from thromboembolic events [1113].

Two of the most widely used mechanical heart valves are ST Jude medical and ATS valves, recent comparative non randomized studies have shown no significant differences between these two (ST Jude and ATS) mechanical prostheses $[10,11]$, however, some have reported that ST Jude mechanical valves perform less efficiently in regard to thromboembolic complications than do ATS prosthesis $[5,1$, 3]. Few randomized studies have compared early and midterm outcomes for ST Jude and ATS heart valves in the mitral position alone $[2,9,16]$.

The intra operative and early postoperative performance of the two type valves was similar, the hospital mortality reported here was lower in ATS group but this difference did not reach statistical significance.

Thromboembolism and anticoagulant related bleeding remain the major impediments to successful use of mechanical valve prosthesis therefore, they are the most specific measures of valve performance [14, 15]. Anticoagulation management is the major determinant of outcome, some studies found the ST Jude mechanical valve to be significantly associated with thromboembolism specially in mitral position [7,8] but in our study, despite some valve thrombosis detected in the ST Jude group, we found no statistically significant difference in freedom of thromboembolism in either group. The average International 
Normalize Ratio (INR) levels in this study were $2,4+-0,8$ and 2,6+- 1,2I both aortic and mitral position in ST Jude and ATS groups.

Disparities in results indicate that the specific characteristics of each patient and the method of anticoagulation may play a greater role in thromboembolism than does the type of mechanical prosthesis.

\section{Conclusion}

Anticoagulant related hemorrhage is another major problem in patients who receive mechanical heart valves specially in our country (AFGHANISTAN) due to lack of patients knowledge regarding anticoagulant medicine and its complications, despite guiding the patients after valve replacement some of them misuse the medicine and have to return to the hospital with thromboembolism events, in evaluating aortic and mitral valve replacement with ST Jude and ATS mechanical valves we detected no late survival advantage of one type of valve over the other one.

Our result indicates that the standard ST Jude and ATS prostheses offer similar excellent clinical performance in both aortic and mitral positions. There is a different of prosthetic valve noise between ATS and ST JUDE mechanical heart valves, signifying that the ATS is better than ST JUDE.

\section{Acknowledgements}

We thank Mr. Ismail NOORI (Chair, department of Anesthesiology) and Mr. Alim ALIMI (Director of Perfusion) for their expertise, contribution and assistance throughout all aspects of this study.

\section{References}

[1] Gott VL, Alejo DE, Cameron DE. Mechanical heart valves: 50 years of evolution. Ann Thorac Surg 2003; 76: S2230-9.

[2] Akins CW. Results with mechanical cardiac valvular prostheses. Ann Thorac Surg 1995; 60: 1836-44.

[3] Edmunds LH Jr, Clark RE, Cohn LH, Grunkemeier GL, Miller DC, Weisel RD. Guidelines for reporting morbidity and mortality after cardiac valvular operations. The American Association for Thoracic Sugery, Ad Hoc Liaison Committee for Standardizing Definitions of Prosthetic Heart Valve Morbidity. Ann Thorac Surg 1996; 62: 932-5.
[4] Sezai A, Shiono M, Orime Y, et al. Evaluation of valve sound and its effects on ATS prosthetic valves in patients' quality of life. Ann Thorac Surg 2000; 69: 507-12.

[5] Gao G, Wu Y, Grunkemeier GL, Furnary AP, Starr A. Fortyyear survival with the Starr-Edwards heart valve prosthesis. J Heart Valve Dis 2004; 13: 91

[6] Tsukamoto S, Shiono M, Orime Y, et al. Macroscopic aspects of cloth-covered Starr-Edwards prostheses at reoperation: what the precursory valve teaches us. J Heart Valve Dis 1998; 7: $556-60$.

[7] Sezai Y. Experience with the St. Jude Medical prosthesis. In: DeBakey ME ed.; Advances in Cardiac Valves: Clinical Perspectives. USA: Yorke Medical Books, 1983; pp 88-102.

[8] Sezai A, Shiono M, Akiyama K, et al. Evaluation of St. Jude Medical valve's long-term function by Doppler echocardiography. Ann Thorac Cardiovascular Surg 2001; 7: 216-22.

[9] Arom KV, Nicoloff DM, Kersten TE, Northrup WF 3rd, Lindsay WG, Emery RW. Ten years' experience with the St. Jude Medical valve prosthesis. Ann Thorac Surg 1989; 47: $831-7$.

[10] Kratz JM, Crawford FA Jr, Sade RM, Crumbley AJ, Stroud MR. St. Jude prosthesis for aortic and mitral valve replacement: a ten-year experience. Ann Thorac Surg 1993; 56: $462-8$.

[11] Nakano K, Koyanagi H, Hashimoto A, et al. Twelve years' experience with the St. Jude Medical valve prosthesis. Ann Thorac Surg 1994; 57: 697-703.

[12] Fernandez J, Laub GW, Adkins MS, et al. Early and late-phase events after valve replacement with the St. Jude Medical prosthesis in 1200 patients. J Thorac Cardiovascular Surg 1994; 107: 394-407.

[13] Zellner JL, Kratz JM, Crumbley AJ 3rd, et al. Longterm experience with the St. Jude Medical valve prosthesis. Ann Thorac Surg 1999; 68: 1210-8.

[14] Hasegawa M. Clinical evaluation of ATS prosthetic valve by Doppler echocardiography: comparison with St. Jude Medical (SJM) valve. Ann Thorac cardiovascular Surg 2000; 6: 24751.

[15] Tayama E, Feng Z, Oda T, et al. ATS prosthetic valve motion: an in vitro analysis. J Heart Valve Dis 2000; 9: 408-14.

[16] Murday AJ, Hochstitzky A, Mansfield J, et al. A prospective controlled trial St. Jude versus Starr Edwards aortic and mitral valve prostheses. Ann Thorac Surg 2003; 76: 66-74. 28. 